\title{
Prospects for Contact Lens Wear in Aphakia
}

\author{
C. M. GRAHAM ${ }^{*}$ J. K. G. DART,${ }^{*} \dagger$ N. W. WILSON-HOLT, ${ }^{*}$ and R. J. BUCKLEY ${ }^{*}$ \\ London
}

\begin{abstract}
Summary
The success rate and complications for contact lens wear in 366 aphakic patients were evaluated retrospectively over a mean follow-up period of 36 months. Successful use of a daily wear contact lens (DWCL) was related to lens handling ability. In unilateral aphakia it was 86 per cent $(161 / 187)$ for patients under 70 years old but only 27 per cent $(19 / 70)$ for those over 70 years $(p=<0.0001)$. This age related difference did not occur either for the use of a second lens, following successful use of a lens after the first cataract extraction, for which the overall success rate was 89 per cent $(\mathbf{5 5 / 6 2})$ or for simultaneous bilateral lens fitting, which had a success rate of 81 per cent (38/47). Extended wear soft contact lenses (EWSCL) were fitted to patients unable to use DWCLs but only half were successful (21/40). There was no difference in the incidence of complications between daily wear soft and daily wear hard contact lenses or young (up to 44 years), middle aged (45-69 years) and elderly (over 70 years) patients, for the use of all DWCLs. However the risk of a serious complication was six times greater for patients using EWSCL ( 55 per cent) compared with those using DWCLs (8.8 per cent). DWCLs are a safe and successful method of aphakia correction for patients under 70 years of age. Once the ability to handle a DWCL has been learned, success is maintained when the second eye is fitted. EWSCLs have a high complication rate and a low success rate in patients unable to use DWCLs; other methods of aphakia correction should be considered first in this group.
\end{abstract}

Although an intraocular lens implant (IOL) is the treatment of choice for most aphakes alternative forms of correction will always be needed for some categories of cataract patient. Because of the good quality of vision obtained the contact lens (CL) is a commonly used alternative for these patients. Common indications for an aphakic CL at Moorfields Eye Hospital are for patients whose surgeon did not or does not practice IOL implantation or because of a surgical or medical contraindication to it. There is little information available on the success and complications of $\mathrm{CL}$ correction in these patients on which to base such decisions on the management of aphakia; the available literature favours the use of the extended wear soft contact lens (EWSCL) rather than the daily wear contact lens (DWCL) in aphakia.

In a recent study, comparing the success and complications for daily wear hard contact lenses (DWHCL) and EWSCLs in comparable groups of elderly aphakic patients, we reported a high failure rate for the use of both lens types and a high risk of complications in the EWSCL group. ${ }^{1}$ The present study retrospectively evaluated a larger group of aphakic patients of all ages using DWHCLs, daily wear soft contact lenses (DWSCL) and EWSCLs to assess the long-term success rates

* The Contact Lens and Prosthesis Department and †The Department of Clinical Ophthalmology, Institute of Ophthalmology, Moorfields Eye Hospital, City Road, London EC1V 2PD.

Correspondence to: J. K. G. Dart, MA, FRCS, Moorfields Eye Hospital, City Road, London EC1V 2PD. 
for these different CLs, whether success was age or lens related, and whether the use of CLs in bilateral aphakia decreased the rate of success. The complications associated with the different lens types were also compared.

\section{Patients, Materials and Methods \\ Patients}

All patients referred for contact lens fitting, to a special aphakia clinic, in 1983 were included. The success of contact lens wear and the outcome of the failures were evaluated for three categories; patients fitted with lenses for unilateral aphakia, patients fitted for bilateral aphakia at their first visit, and patients who were either having their second eye fitted after a period of successful contact lens wear on the first eye or whose first eye was fitted in 1983 and who subsequently became bilaterally aphakic. Complications were assessed for all the eyes of those patients fitted in 1983; complications occurring in eyes fitted before and after 1983 were not assessed. The follow-up range was 0-49 months (mean 35.9).

The function of the aphakia clinic, in which these patients were seen, has been described in detail. ${ }^{2.3}$ Patients are only seen in this clinic if their refractive error falls within specified limits, if they have no ocular abnormality other than aphakia and if no surgical complication has occurred that might affect contact lens management. Seventy per cent of patients could be fitted with a stock lens on their first visit to the clinic. The remainder could not be fitted with a stock lens at all; these were mainly those with corneal astigmatism requiring a different lens form. These patients were excluded from the comparison so that only one type each of DWHCL and EWSCL, and two types of DWSCL were used in the study group. Patients fitted with DWCLs were instructed at their first visit and reviewed at 1 month, 3 months, 6 months and then yearly. EWSCL users were seen more often initially and later at intervals of no more than 3 months for removal, cleaning, disinfection and replacement. New lenses were issued after damage or loss. Prophylactic antibiotics were not used. Patients who had complications were seen by an optometrist and an ophthalmologist in the Contact Lens Department during working hours or, at other times, in the Accident and Emergency Department of the hospital, by the resident surgical officer on call.

Table Ia shows the numbers of patients in each of the three age groups used in the analysis, the losses of follow-up and the follow-up range and mean.

\section{Lenses}

The first choice of CL for all patients was a DWHCL, (Titmus Eurocon Persecon E lens, Titmus Eurocon Ltd, Portsmouth, UK) made from cellulose acetyl butyrate with an elliptical back lens form. These were available in an overall diameter (OD) of $9.8 \mathrm{~mm}$ with back central optic radii (BCOR) of 7.5 to $8.2 \mathrm{~mm}$ and with back vertex powers (BVD) from $+13 \mathrm{D}$ to $+18 \mathrm{D}$. A few DWSCLs were fitted, mainly to overcome difficulty in achieving centration with the DWHCL. These were made in hydroxy-ethyl methacrylate and were lathe cut or spun-cast. The lathe cut lenses (Hydron Europe Ltd, Camberley, UK) were available in ODs 13 to $14 \mathrm{~mm}$, BCORs 8.1 to $9.1 \mathrm{~mm}$ and BVDs +13D to +18D. The spuncast lenses (Bausch and Lomb UK Ltd, Hampton, UK) had ODs 13.5 to $14 \mathrm{~mm}$ and BVDs +12D to +20D. EWSCLs were only

Table Ia Patient numbers, losses to follow-up by age group and follow-up period

\begin{tabular}{lcccc}
\hline & \multicolumn{4}{c}{ Age groups } \\
\cline { 2 - 5 } & \multicolumn{1}{c}{$<0-44$} & $45-69$ & $70->80$ & Total \\
\hline Patient numbers & 57 & 228 & 89 & 374 \\
Lost to follow-up & 2 & 2 & 8 & 366 \\
Number in analysis & 55 & 226 & 85 & Range 0-49 months \\
Follow-up period & \multicolumn{5}{c}{ Mean 35.9 months } \\
\hline
\end{tabular}


fitted to patients unable to handle a DWCL. The EWSCL used was lathe cut in the Contact Lens Department from Duragel 75 (Coopervision UK, Southampton, UK), a complex polymer of methacrylate and vinyl pyrollidone, with a fully hydrated water content of 75 per cent. It was available in ODs 14 to $14.5 \mathrm{~mm}$, BCORs 7.8 to $8.7 \mathrm{~mm}$ and BVDs $+12 \mathrm{D}$ to $+20 \mathrm{D}$.

\section{Statistical analysis}

The 95 per cent confidence limits of percentages were calculated from the standard errors. The Chi square test was used for null hypothesis testing of proportional differences between groups and the confidence limits for relative risk values calculated using Miettinen's test-based approximate confidence limits. ${ }^{4}$

\section{Results}

Success

Success was defined as regular use of the $\mathrm{CL}$ throughout the study period. Table Ib shows the relationship of success with age for DWCLs used in the three categories of patient. Two hundred and fifty seven patients were fitted unilaterally with a DWCL during the study period (242 DWHCL and 15 DWSCL). Of the remaining 109 patients, 47

Table Ib Patients in each of three lens wearing categories by age group showing total numbers and proportion successful with the analysis of the success rate for different age groups

\begin{tabular}{|c|c|c|c|c|}
\hline \multirow[b]{2}{*}{ Lens wearing category } & \multicolumn{3}{|c|}{ Number in each age group } & \multirow[b]{2}{*}{ Total } \\
\hline & $\begin{array}{l}<20-44 \\
\text { years }\end{array}$ & $\begin{array}{l}45-69 \\
\text { years }\end{array}$ & $\begin{array}{l}70->80 \\
\text { years }\end{array}$ & \\
\hline \multicolumn{5}{|l|}{ aUnilateral ${ }^{\mathrm{b}} \mathrm{DWCL}$} \\
\hline All users & $39(5)$ & $148(6)$ & $70(4)$ & 257 \\
\hline Successful users & $36(4)$ & $125(4)$ & $19(2)$ & 6180 \\
\hline Proportion successful & $92 \%$ & $84 \%$ & $27 \%$ & $70 \%$ \\
\hline \multicolumn{5}{|l|}{$\begin{array}{l}\text { Difference in success between }<20-44 \\
\text { and 45-69: cNS }(p=0.2)\end{array}$} \\
\hline \multicolumn{5}{|l|}{$\begin{array}{l}\text { Difference in success between }<20-69 \\
\text { and } 70->80 \text { : }\end{array}$} \\
\hline$\%$ success & \multicolumn{2}{|c|}{$86 \%$} & \multicolumn{2}{|l|}{$27 \%$} \\
\hline $95 \%$ confidence limits $(\mathrm{p}=<0.0001)$ & \multicolumn{2}{|c|}{$81 \%-91 \%$} & \multicolumn{2}{|l|}{$17 \%-37 \%$} \\
\hline \multicolumn{5}{|l|}{ Bilateral DWHCL wear } \\
\hline All users & 7 & 34 & 6 & 47 \\
\hline Successful users & 5 & 28 & 5 & 38 \\
\hline Proportion successful & $71 \%$ & $82 \%$ & $83 \%$ & $81 \%$ \\
\hline \multicolumn{5}{|l|}{$\begin{array}{l}\text { Difference in success between age groups: } \\
\text { NS }(p=0.97)\end{array}$} \\
\hline \multicolumn{5}{|l|}{ Success overall groups: } \\
\hline$\%$ success & & & & $81 \%$ \\
\hline $95 \%$ confidence limits & & & & $70 \%-92 \%$ \\
\hline \multicolumn{5}{|l|}{$\begin{array}{l}\text { dSecond lens worn after successful use of } \\
\text { lens on first aphakic eye }\end{array}$} \\
\hline All users & 9 & 44 & 9 & 62 \\
\hline Successful users & 8 & 39 & 8 & 55 \\
\hline Proportion successful & $89 \%$ & $89 \%$ & $89 \%$ & $89 \%$ \\
\hline \multicolumn{5}{|l|}{ Success overall groups: } \\
\hline$\%$ success & & & & $89 \%$ \\
\hline $95 \%$ confidence limits & & & & $81 \%-97 \%$ \\
\hline
\end{tabular}

aMost patients used daily wear hard contact lenses. The numbers in brackets indicate the patients using the daily wear soft contact lenses.

bDaily wear contact lens (hard or soft).

cNot significant.

'Only 14 of these fitted in 1983, all of whom were successful; remainder have a shorter follow up. 
patients were referred for bilateral contact lens fitting at their first visit, and 62 had a second lens fitted after successful use of an aphakic CL following their first cataract extraction. Fourteen of these were wearing their first CL prior to the fitting of their second lens in 1983, and 48 had their first eye successfully fitted in 1983 and returned for fitting of their second eye during the remaining period of follow-up.

Unilateral DWCL success was strongly related to age and lens handling ability. There was no difference in the success rate $(p=0.207)$ between patients aged up to 44 and 45-69, in whom there was an overall success of 86 per cent. However the success rate fell to 27 per cent for patients aged 70 and over, a significant difference $(p=<0.0001)$. The main reason for failure overall was difficulty handling the lenses (64/77 failures). The remaining failures were due to lens intolerance in six, failure to improve the vision in four, corneal neovascularisation in two and recurrent corneal erosion in one. Over the age of seventy 96 per cent of the patients failed due to handling inability (49/51). The success and lens handling ability was similar in the 15 DWSCL users as in the 242 DWHCL users.

Bilateral $D W C L$ patients were all fitted bilaterally at their first visit. No DWSCLs were fitted in this group. The success rate was
81 per cent overall and there was no difference in the success with age. Handling difficulties were responsible for six of the nine failures. The remaining three failures were due to lens intolerance in two and corneal oedema in one.

DWCL on second eye after successful DWCL use on first eye. No DWSCLs were fitted in this group. The overall success rate was 89 per cent and was not affected by age. All seven failures preferred to use an aphakic spectacle correction because of difficulty in handling two lenses.

Twelve patients who were wearing a DWCL successfully when they had primary intraocular lens implantation in their second eye continued to use their unilateral CL.

\section{Outcome}

Table Ic shows the outcome for the 93 DWCL failures, including those who attempted EWSCL wear. Fifty-six of these patients eventually used aphakic spectacles and only seven required a secondary IOL implant. Nine patients abandoned any form of correction for their aphakic eye and of the 40 patients who attempted EWSCL use only 21 continued to the end of the study.

\section{Complications}

Table IIa shows the number of episodes of complications that occurred in the eyes fitted

Table Ic Outcome for daily wear contact lens failures and extended wear soft contact lens users

\begin{tabular}{|c|c|c|c|c|}
\hline \multirow[b]{2}{*}{ Patient category } & \multicolumn{3}{|c|}{ Age groups } & \multirow[b]{2}{*}{ Total } \\
\hline & $<20-44$ & $45-69$ & $70->80$ & \\
\hline \multicolumn{5}{|l|}{ Outcome of failed lens wearers } \\
\hline All failures & 6 & 34 & 53 & 93 \\
\hline No correction & 2 & 3 & 2 & 7 \\
\hline Glasses & 2 & 24 & 15 & 41 \\
\hline Secondary aIOL implantation & 2 & 3 & 0 & 5 \\
\hline 'EWSCL & 0 & 4 & 36 & 40 \\
\hline \multicolumn{5}{|l|}{ Outcome of EWSCL users } \\
\hline All users & & 4 & 36 & \\
\hline Successful users & \multicolumn{4}{|c|}{21} \\
\hline No correction & \multicolumn{4}{|c|}{2} \\
\hline Glasses & \multicolumn{4}{|c|}{15} \\
\hline Secondary IOL implantation & \multicolumn{4}{|c|}{2} \\
\hline
\end{tabular}

antraocular lens.

bxtended wear soft contact lens. 
in 1983, the number of individual eyes that these episodes affected, and the number of patients who had no complication at all. Complications were divided into major, serious and minor. Major complications were sight threatening, of which suppurative keratitis was the only one recorded. Serious complications were those which resulted in the loss of lens wearing time for at least 1 week. Minor complications were observed at follow-up visits but did not require any treatment that altered the patients' patterns of lens wear.

Table IIb summarises the comparison of complications relating to the different age groups and lens types. There was no overall difference between the incidence of all complications for DWHCL and DWSCL $(p=0.9)$. Although the trend was for a higher proportion of the serious complications to occur in the over 70 age group this was not significant at the 5 per cent level $(\mathrm{p}=0.30)$. There was a great difference between the incidence of complications in DWCL and EWSCL eyes. These groups have different age ranges, so are not strictly comparable. Major and serious complications occurred in 8.8 per cent of DWCL eyes and 55 per cent of EWSCL eyes, a difference in incidence of 46

Table IIa a Complications of contact lenses in 413 eyes of 366 patients

\begin{tabular}{|c|c|c|c|c|c|c|c|c|}
\hline \multirow{3}{*}{$\begin{array}{l}\text { Complication group } \\
\text { Follow-up period } \\
\text { Range } 0-49 \text { months } \\
\text { Mean } 35.9 \text { months }\end{array}$} & \multicolumn{8}{|c|}{$\begin{array}{l}{ }^{\mathrm{b}} \text { Number of complication episodes in affected eyes } \\
\text { occurring by age and lens type }\end{array}$} \\
\hline & \multicolumn{2}{|c|}{$<20-44$ years } & \multicolumn{3}{|c|}{$45-69$ years } & \multicolumn{3}{|c|}{$70->80$ years } \\
\hline & \multicolumn{2}{|c|}{${ }^{\mathrm{c}} D W H C L D W S C L$} & $W H C$ & WSC & $V S C$ & NHC & $W S C L$ & $V S C L$ \\
\hline \multirow{2}{*}{$\begin{array}{l}\text { Major } \\
\text { Microbial keratitis }\end{array}$} & & & & & & & & \\
\hline & - & - & - & - & 1 & - & - & 2 \\
\hline Eyes & - & 一 & - & - & 1 & - & - & 2 \\
\hline \multicolumn{9}{|l|}{ Serious $\quad$ Episodes } \\
\hline Tight fit syndrome & - & - & - & - & - & - & - & 2 \\
\hline Striae & - & - & 4 & 1 & - & - & 1 & 3 \\
\hline Corneal erosion & 2 & - & 13 & - & - & 3 & 1 & 5 \\
\hline dNeovascularisation & 1 & - & 2 & - & - & 1 & - & 3 \\
\hline Stromal oedema & 2 & - & 4 & 1 & 2 & 2 & - & 9 \\
\hline Blepharoconjunctivitis & - & - & 4 & 1 & - & - & - & 6 \\
\hline Eyes & 4 & - & 21 & 1 & 2 & 6 & 1 & 17 \\
\hline \multicolumn{9}{|l|}{ Minor $\quad$ Episodes } \\
\hline Punctate keratopathy & 27 & 2 & 84 & 2 & - & 17 & 2 & 5 \\
\hline Blepharitis & 5 & - & 10 & 1 & - & 1 & - & 一 \\
\hline Epithelial oedema & 10 & 1 & 33 & 3 & - & 1 & 1 & - \\
\hline Neovascularisation & 2 & - & 3 & 1 & - & 4 & - & 1 \\
\hline eGPC & 4 & - & 13 & - & - & - & - & 2 \\
\hline Epithelial microcysts & 3 & - & 10 & 一 & 一 & - & - & 2 \\
\hline Eyes & 30 & 2 & 101 & 6 & - & 18 & 2 & 6 \\
\hline Eyes without complications & 21 & 5 & 122 & 5 & 1 & 27 & 1 & 11 \\
\hline
\end{tabular}

aEyes fitted before entry into study and after 1983 not included.

bEyes are classified by their most severe complication so that minor complications are not recorded for eyes with a serious complication.

'DWHCL - daily wear hard contact lens, DWSCL—daily wear soft contact lens, EWSCL-extended wear soft contact lens.

dNeovascularisation with $>2 \mathrm{~mm}$ extension into cornea.

'Giant papillary conjunctivitis (includes early cases without 'giant' papillae). 
Table IIb Summary of complications

Comparison of complications in aWHCL and ${ }^{\mathrm{b}} \mathrm{DWSCL}$.

All age groups combined

No significant difference $\left(\mathrm{X}^{2}=0.0072 \mathrm{P}=0.9\right)$

Comparison of complications in 'DWCL by age group $<20-44,45-69$ and $70->80$

No significant difference $\left(\mathrm{X}^{2}=4.1 \mathrm{P}=0.3\right)$

Comparison of DWCL and ${ }^{\mathrm{d}} \mathrm{EWSCL}$ for serious and major complications:

$\%$ complications

$95 \%$ confidence limits

Relative risk

Incidence difference

$\mathrm{X}^{2}$

$\mathrm{P}$
DWCL
$8.8 \%$
$6 \%-11.7 \%$

EWSCL
$55 \%$

$39.6 \%-70.4 \%$

$95 \%$ confidence limits

6.2
$46 \%$
66.66
$<0.0001$

Comparison of DWCL and EWSCL for all complications:

$\%$ complications

$95 \%$ confidence limits

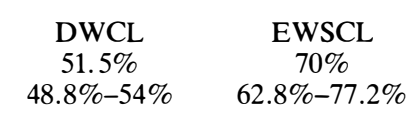

$95 \%$ confidence limits

Relative risk

Incidence difference

$\mathrm{X}^{2}$

$\mathrm{P}$
1.36

$18.5 \%$

67.33

$<0.0001$

aDaily wear hard contact lens.

bDaily wear soft contact lens.

cDaily wear contact lens.

dExtended wear soft contact lens.

per cent $(p=<0.0001)$. The risk of developing this category of complication in an EWSCL eye was six times greater than with a DWCL. These differences were less if all complications, including the minor complications, of which punctate keratopathy was very common in DWCLs, were compared. Fifty two per cent of DWCL eyes had a recorded minor complication compared to 70 per cent for EWSCL eyes; an incidence difference of 18.5 per cent $(p=0.0001)$. The risk of developing any complication was 1.36 times higher for EWSCL wear.

\section{Discussion}

For most elderly patients primary implantation of a posterior chamber IOL (PC-IOL) is the preferred method of aphakia correction and is, in the short term, associated with few complications. Serious complications do occur, however, particularly with other types of IOL, and there is less than 12 years' follow-up of the modern PC-IOLs. ${ }^{5}$ The significance of subclinical long term endothelial cell loss with the posterior chamber and other IOLs, ${ }^{6.7}$ the breakdown of the blood-aqueous barrier with anterior chamber IOLs and sulcus fixated PC-IOLs ${ }^{8}$ and experimental evidence of activation of the alternative complement pathway by components of flexible loop IOLs ${ }^{9}$, is uncertain. But these findings must raise some doubts about the advisability of IOL implantation in young aphakic patients. Although there is no consensus on the contraindications to primary IOL implantation, anterior uveitis, a disorganised anterior segment, proliferative diabetic reti- 
nopathy, and per-operative complications are additional states in which an alternative method of aphakia correction must be considered.

Currently a contact lens will provide high quality vision without the need for further surgery in these groups of patients. The DWHCL has been in use for several decades without any serious concern about its longterm complications, although CLs, like IOLs, are not without long-term ocular effects, ${ }^{10,11}$ also of uncertain significance. Unlike the IOL the CL can be easily changed or updated and has the additional advantage that it does not preclude the use of any other form of aphakic correction if it is not successful. A knowledge of the success and complication rates of contact lens correction for aphakia is important so that the management of all aphakic patients can be optimised.

In our series there was a success rate of 86 per cent in patients under 70 years of age for unilateral DWCL wear. This figure fell to 27 per cent in those over 70 because of difficulty in handling the lenses. It is surprising that the change in dexterity altered so markedly at this age. The inability to handle a DWCL was not seen in the few patients over 70 fitted with two lenses at the same time, probably because these patients were more highly motivated after an unsuccessful period of spectacle aphakia correction; overall the success rate was 81 per cent in this group. In the small number of patients over 70 years who were fitted with a DWHCL following their second cataract extraction, after satisfactory use of a lens for their first eye, only 11 per cent failed to use both lenses satisfactorily ( 89 per cent success). This shows that a high success rate can be maintained for CL wear in bilateral aphakia. The incidence of serious complications resulting in a loss of wearing time was 8.8 per cent for DWCLs. When DWHCLs were compared with the small number of DWSCLs there was no apparent difference in success nor a significant difference in the incidence of complications.

Only patients failing to handle a DWCL were fitted with an EWSCL, mostly in the over 70 year age group, and the success rate remained low at 52 per cent. This type of lens was associated with a high incidence of com- plications, a serious complication occurring in 55 per cent of EWSCL eyes, six times higher for EWSCL as opposed to DWSCL. It is possible that this increased risk of complications found in the EWSCL group is related to the increased age of the patients using this lens type; this is unlikely, however, as our previous study of two age matched groups using DWHCL and EWSCL, showed that the risk of a serious complication was at a similar level, at 11.4 times (95 per cent confidence limits 4.1 times to 31.7 times). ${ }^{1}$ In addition the current study has shown no significant increase in the incidence of complications with age in the DWCL group.

There are few reports of the success of DWHCL in aphakia. Our success rate for patients under 70 with uniocular aphakia was better than in an early comparison of polymethylmethacrylate CLs with IOLs in which only 8/25 (32 per cent) were successful. ${ }^{12}$ Later studies have investigated the success and complications of aphakic EWSCLs. The success rate was higher in these studies than in our more elderly group of EWSCL users, ranging from 78 per cent over 6 months ${ }^{13}$ to 83 per cent at 23 months $^{14}$ - similar to our results for DWCLs in the under 70 age group. One prospective randomised controlled trial showed no difference in the success or complications of aphakia EWSCL compared with an iris clip IOL. ${ }^{15}$ The Oxford Cataract Treatment and Evaluation Team has recently reported a controlled trial, with a prolonged follow-up, showing no difference in the success and complications for intracapsular cataract extraction and CL wear compared with extracapsular extraction with an iridocapsular lens. Both these methods of correction had significantly fewer complications than intracapsular extraction with an iris clip IOL. Their success rate for CL wear was 89 per cent-within the 95 per cent confidence limits for all our patient categories. ${ }^{16.17}$

The high success rate for DWCL, in the unilateral and bilateral aphakic patient under 70 years in this series, together with the low risk of a serious complication compared to EWSCL use, supports our policy for fitting a DWCL as first choice and demonstrates that the DWCL remains a viable alternative to the IOL in this group of patients. In the patient 
over 70 years the success rate for all types of contact lens is low and the complications of EWSCL high. An aphakic spectacle correction should be tried before CL wear in these patients if lens implantation is contraindicated.

The authors thank C. Astin, FBCO, DCLP, for supplying the list of patients seen in the aphakia clinic.

\section{References}

${ }^{1}$ Graham CM, Dart JKG, Buckley RJ: Extended wear hydrogel and daily wear hard contact lenses for aphakia. Success and complications compared in a longitudinal study. Ophthalmology 1986, 93: 1489-94.

${ }^{2}$ Astin C: Aphakia contact lens fitting in a hospital department. J Br Contact Lens Assoc 1984, 7: 164-8.

${ }^{3}$ Astin C: Review of the 2 nd year of the aphakic clinic at Moorfields Eye Hospital contact lens department. J Br Contact Lens Assoc 1986, 9 (Suppl): 14-8.

${ }^{4}$ Miettinen OS: Simple interval estimation of risk ratio. Am J Epidemiol 1974, 100: 515-6.

${ }^{5}$ Apple DJ, Mamalis N, Loftfield K, Googe JM, Novak LC, Kavka-Van Norman D, Brady SE, Olson RJ: Complications of intraocular lenses. A historical and histopathological review. Surv Ophthalmol 1984, 29: 1-54.

${ }^{6}$ Liesegang TJ, Bourne WM, Ilstrup DM: Short- and long-term endothelial cell loss associated with cataract extraction and intraocular lens implantation. Am J Ophthalmol 1984, 97: 32-9.

${ }^{7}$ Oxford Cataract Treatment and Evaluation Team (OCTET): Long-term corneal endothelial cell loss after cataract surgery. Results of a random- ised controlled trial. Arch Ophthalmol 1986, 104: $1170-5$.

${ }^{8}$ Miyake K, Akasura M, Kobayashi H: Effect of intraocular lens fixation on the blood-aqueous barrier. Am J Ophthalmol 1984, 98: 451-5.

${ }^{9}$ Mondino BJ, Nagata S, Glovsky MM: Activation of the alternative complement pathway by intraocular lenses. Invest Ophthalmol Vis Sci 1985, 26: 905-8.

${ }^{10}$ Holden BA, Sweeney DF, Vannas A, Nilsson KT, Efron N: Effects of long-term extended contact lens wear on the human cornea. Invest Ophthalmol Vis Sci 1985, 26: 1489-501.

${ }^{11}$ Mac Rae SM, Matsuda M, Shellans S, Rich LF: The effects of hard and soft contact lenses on the corneal endothelium. Am J Ophthalmol 1986, 102: $50-7$.

12 Percival SPB and Yousef KM: Treatment of uniocular aphakia. A comparison of iris clip lenses with hard corneal contact lenses. Br J Ophthalmol 1976, 60: 642-4.

${ }^{13}$ Kersley HJ, Kerr C, Pierse D: Hydrophilic lenses for 'continuous' wear in aphakia: definitive fitting and the problems that occur. Br J Ophthalmol 1977, 61: 38-42.

${ }^{14}$ Martin NF, Kracher GP, Stark WJ, Maumenee AE: Extended-wear soft contact lenses for aphakic correction. Arch Ophthalmol 1983, 101: 39-41.

${ }^{15}$ Bernth-Petersen $\mathrm{P}$ and Sørensen T: Intraocular lenses versus extended-wear contact lenses in aphakic rehabilitation. A controlled clinical study. Acta Ophthalmol 1983, 61: 381-91.

${ }^{16}$ Oxford Cataract Treatment and Evaluation Team (OCTET): I. Cataract surgery: interim results and complications of a randomised controlled trial. Br J Ophthalmol 1986, 70: 402-10.

${ }^{17}$ Oxford Cataract Treatment and Evaluation Team (OCTET). II. Use of a grading system in the evaluation of complications in a randomised controlled trial on cataract surgery. $\mathrm{Br} J$ Ophthalmol 1986, 70: 411-4. 